\title{
Control of cylinder wake flow and noise through a downstream porous treatment
}

\author{
Chen $\mathrm{Xu}^{1,2^{*}}$, Yijun $\mathrm{Mao}^{1,2}$, Zhiwei $\mathrm{Hu}^{2}$ \\ 1 School of Energy and Power Engineering, Xi'an Jiaotong University, 710049 Xi'an, People's Republic of China \\ 2 Faculty of Engineering and Physical Sciences, University of Southampton, SO17 1BJ Southampton, United Kingdom
}

\begin{abstract}
Various passive and active methods have been developed to control flow separation from bluff bodies. However, these methods require adjusting features of the solid surface, such as modifying its geometry or porosity, or applying external force or momentum. This paper develops an off-body-based method, without adjusting any features of the solid surface, for controlling the unsteady flow separation by fixing porous materials downstream of bluff bodies. Numerical study on flow past a circular cylinder at a subcritical Reynolds number is performed, and the result indicates that the added downstream porous material changes flow in the wake and re-laminarizes the turbulent flow around the curved cylinder surface, reducing the wall pressure fluctuation around the cylinder. therefore, the associated aerodynamic noise is reduced greatly.
\end{abstract}

\section{Keywords}

Open-cell metal foam; flow separation; flow control; aerodynamic noise

\section{Nomenclature}

$$
\begin{aligned}
& C_{d r m s}=\text { root-mean square of drag coefficient } \\
& \bar{C}_{d}=\text { time-averaged drag coefficient } \\
& C_{l r m s}=\text { root-mean square of lift coefficient } \\
& \bar{C}_{l}=\text { time-averaged lift coefficient }
\end{aligned}
$$

*Corresponding author. xuchen1983@xjtu.edu.cn; c.xu@ soton.ac.uk (Chen Xu). 


$$
\begin{aligned}
& D=\text { diameter of the circular cylinder } \\
& d_{p} \quad=\text { mean particle diameter } \\
& r \quad=\text { distance between source and observer } \\
& U \quad=\text { instantaneous velocity of local flow } \\
& U_{\infty} \quad=\text { mean flow velocity } \\
& \bar{U}_{x} \quad=\text { x-direction component of time-averaged flow velocity } \\
& \bar{U}_{y}=\text { y-direction component of time-averaged flow velocity } \\
& S t \quad=\quad \text { Strouhal number } \\
& \operatorname{Re}=\text { Reynolds number } \\
& \varepsilon \quad=\text { porosity } \\
& \mu \quad=\text { dynamic viscosity } \\
& v \quad=\text { kinematic viscosity }
\end{aligned}
$$




\section{Introduction}

Phenomena of vortices shedding from bluff bodies widely exist in various engineering applications. Usually, these phenomena cause a significant increase in the time-averaged flow drag due to the pressure drop at the rear surface of the body. Moreover, these phenomena also result in a notable increase in lift and drag fluctuations as well as associated vibration and noise. Therefore, flow separation control is of great importance for many applications of fluid mechanics.

Flow control methods have been explored for decades, and these methods can be classified into active control and passive control. The active control is based on influencing the flow by exerting external force or momentum, such as plasma actuators, injection and suction of fluids. The passive control is usually through modifications of bluff body geometry, such as serrations and splitter plates, to affect the formation of vortex shedding.

Many studies on active and passive flow controls have been performed and comprehensive review can be found in [1-9].Therefore, only some representative studies are mentioned below. Active flow control, generates a secondary flow through, such as injection and suction of fluid. Control of turbulent boundary layers around a cylinder by using surface suction was performed experimentally by Seal and Smith [10], and the result showed that the surface suction can weaken both the instantaneous vortex-surface interaction and the downstream extension of the turbulent necklace vortex. Kim and Choi [11] applied a distributed forcing to flow over a circular cylinder for drag reduction, and the distributed forcing was realized by blowing and suction from slots placed at both the upper and lower surfaces of the cylinder. Tensi et al. [12] studied experimentally on controlling flow around an obstacle by means of unsteady blowing through a single slot disposed on the surface of the obstacle, in order to investigate the effect of the jet 
injection on delaying separation and modifying the drag coefficient. Liu and Feng [13] investigated numerically to suppress lift fluctuations and to reduce the mean drag of a circular cylinder by using synthetic jets.

Another promising active method for controlling flow is the excitation of plasma actuators. Post and Corke [14] applied plasma actuators to control flow separation from an airfoil. Two types of actuators were placed at the leading edge of the airfoil, one produced a spanwise array of streamwise vortices, and another produced a two-dimensional jet in the streamwise direction. The result showed that reattached flow created by the plasma actuators can result in a decrease in drag and suction-pressure recovery. Nati et al. [15] performed an experimental investigation on controlling of vortex shedding from a blunt trailing edge by fixing plasma actuators in the region of the trailing edge, and demonstrated that plasma actuators can greatly reduce the total kinetic energy due to suppressing vortex shedding from the trailing edge. Recently, Abbas et al. [16] investigated experimentally control of over an airfoil stall by using dual excitations and found that, compared with the approach of using a single plasma actuator at the trailing edge solely, the simultaneous excitation at both the trailing edge and on the suction side was more effective for a full stall control.

On the other hand, passive flow control has also been studies extensively. Splitter plate, as a passive control method, was widely used to suppress vortex shedding and reduce the flow drag. Kwon [17] simulated the effect of splitter plates attached to a circular cylinder on controlling the vortex shedding from a cylinder. The result showed that the vortex shedding behind the circular cylinder completely disappeared when the length of the splitter plate was greater than a critical length, and the critical length was supposed to be proportional to the Reynolds number. Control of flow-induced fluctuating forces on a circular cylinder by means of a splitter plate was also 
numerically investigated by Hwang et al [18]. Results indicated that the splitter plate can suppress the vortex shedding and efficiently reduces drag and lift fluctuations.

For airfoils with a blunt leading and/or trailing edges, wavy or serrated shapes are usually effective to suppress the formation of vortex shedding. Pérez-Torró and Kim [19] performed a large-eddy simulation on a deep-stalled airfoil with a wavy leading edge, revealing that an increased lift and a decreased drag were achieved by using the wavy leading edge compared to the straight leading edge. Additionally, the wavy leading edge significantly reduced the fluctuating component of aerodynamic forces at the frequency of the periodic vortex shedding. Thomareis et al. [20] employed a dynamic mode decomposition to illustrate that the serrated trailing edge of a NACA 0012 airfoil can significantly reduce both the time-averaged and fluctuating components of the drag compared to the blunt trailing edge airfoil, due to attenuation of the turbulence energy by decorrelating the spanwise coherence of the vortices.

Porous material covering on solid surfaces is also classified as a passive flow control method. Gozemen [21] suppressed vortex shedding from a circular cylinder by using a porous coating in deep water, showing that the turbulent intensity of the flow downstream the porous cylinder was much smaller than the rigid solid cylinder. Sueki et al. [22] measured the effect of different types of porous materials on flow around cylinder and the associated noise, it was found that the opencell metal material is effective in delaying the vortex shedding, suppressing pressure fluctuations on the cylinder surface, and reducing aerodynamic noise. Liu et al. [23] numerically studied twodimensional flow around a circular cylinder with coating open-cell metal foams, and analyzed influences of the porosity, pores per inch, thickness of a covering layer on the flow and noise. Results showed that porous material with high porosity can delay the vortex shedding. Liu et al. 
[24] further studied the effectiveness of porous treatment on tandem cylinders to control and regularize the vortex shedding.

Based on the preceding literature review of active and passive flow controls, we can find that all existing flow control methods are achieved by adjusting features of solid surfaces, modifying geometry (splitter plate and serration) or porosity (porous material), or exacting external force (plasma actuators) or momentum (injection and suction). However, some specific engineering applications, do not allow modification of any features of solid surfaces, thus all flow control methods mentioned above cannot be adopted. Therefore, it is meaningful to develop an off-bodybased method for controlling flow separation from the bluff body. In this paper, in order to control flow separation from a circular cylinder at a subcritical Reynolds number, an off-bodybased method, which is achieved by fixing porous materials downstream the cylinder, is developed to suppress the alternating vortex shedding. The developed off-body-based method aims at suppressing unsteady flow separation and reducing aerodynamic noise without changing any features of the solid surface.

The remainder of this paper is organized as follows. Section 2 introduces mathematical methodology, including model description, numerical method and validation. In Section 3, the results are illustrated to analyze the effect of the downstream porous material to suppress the flow separation as well as to control noise. Conclusions are drawn in section 4 . 


\section{Mathematical methodology and validation}

\subsection{Model description}

Two cases are studied numerically to investigate the effect of the off-body porous material on the development of the mean flow and turbulence in the wake. The first case is a classic flow model, i.e., a uniform flow with a freestream velocity of $U_{\infty}=2.18376 \mathrm{~m} / \mathrm{s}$ past a smooth circular cylinder with a diameter of $D=25 \mathrm{~mm}$. The Reynolds number for this case is $R e=3900$, which is in the subcritical Reynolds number range for flow past a circular cylinder. This case has been studied numerically and experimentally by various investigators, such as Norberg [25], Breuer [26] and Meyer [27], thus abundant database can be used to validate the numerical method used in this paper as well as to investigate the feature of the wake development.

In the second case, both the cylinder diameter and free stream velocity are the same as those used in the first case. However, a porous material with a thickness of $10 \mathrm{~mm}(0.4 D)$ is placed downstream of cylinder with the distance of $1.5 \mathrm{D}$ between the center of the cylinder and the porous material. This case aims at investigating the effect of the porous material on developments of both the wake mean flow and turbulence. In the current study, the porosity of the porous material is $96 \%$ and the pore per inch is 40 , which performs best in reducing fan tonal noise as shown by the authors' previous experimental study [28].

The computational domain is $40 D^{*} 20 D^{*} \pi D$ as shown in Fig. 1. The upstream and downstream boundaries are $10 \mathrm{D}$ and $30 \mathrm{D}$ away from the centre of cylinder surface, respectively. A spanwise length of $\pi D$ is chosen according to previous numerical studies of Breuer [26] and Lysenko [29]. A uniform velocity is imposed at the inlet boundary without any perturbation, and a uniform pressure boundary condition is defined at the outlet boundary. No-slip boundary 
condition is applied on the solid wall of the cylinder and periodic boundary conditions are used at the rest pairs of surfaces of the computational domain.

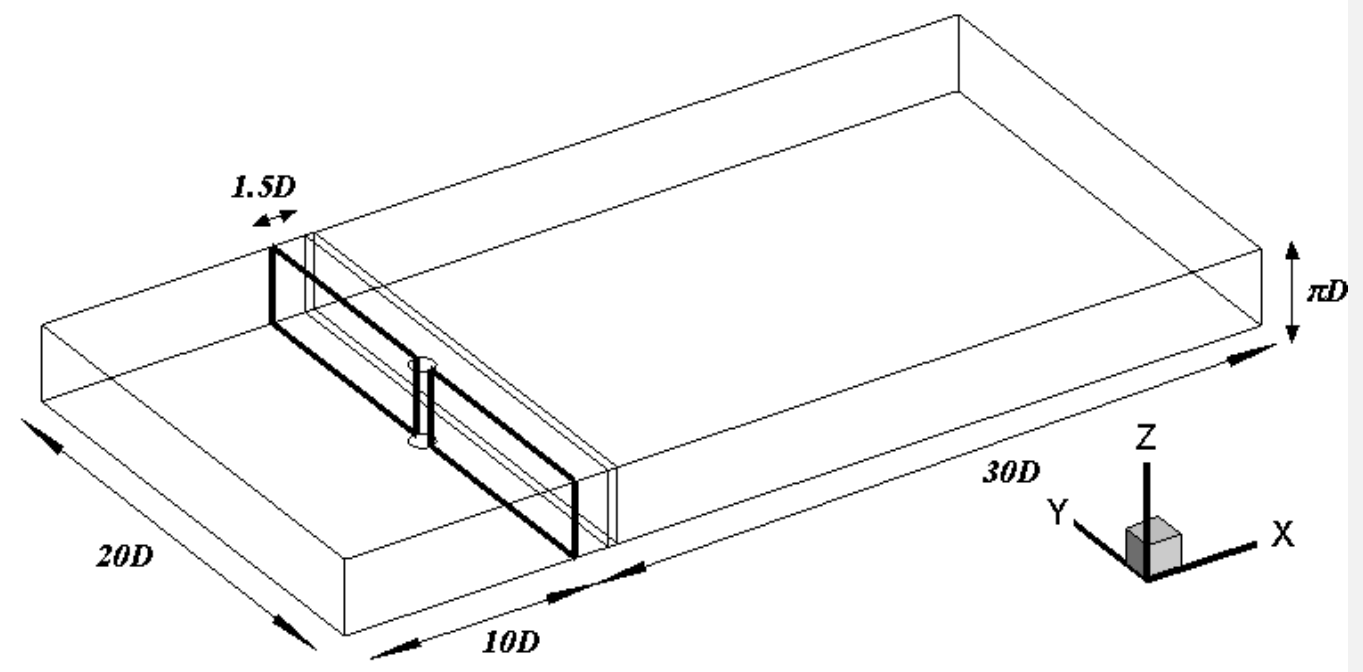

Fig. 1 Outline view of the computational domain for flow simulation

\subsection{Numerical method}

Large eddy simulation (LES) is performed to simulate unsteady features of the flow past the circular cylinder. Outside the porous region, the following filtered incompressible Naiver-Stokes equations are applied to calculate unsteady flow

$$
\begin{gathered}
\frac{\partial \bar{u}_{i}}{\partial x_{i}}=0 \\
\frac{\partial \bar{u}_{i}}{\partial t}+\frac{\partial}{\partial x_{j}}\left(\bar{u}_{i} \bar{u}_{j}\right)=-\frac{1}{\rho} \frac{\partial \bar{p}}{\partial x_{i}}+v \frac{\partial^{2} \bar{u}_{i}}{\partial x_{j} \partial x_{j}}-\frac{\partial \tau_{i j}}{\partial x_{j}} \\
\tau_{i j}=\overline{u_{i} u_{j}}-\bar{u}_{i} \bar{u}_{j}
\end{gathered}
$$

where $\bar{u}_{i}$ is the resolved velocity component, $\bar{p}$ is pressure. $\tau_{i j}$ is subgrid scale stress tensor resulting from filtering the non-linear convective fluxes and describing the influence of the small scale structure on the larger eddies. In the present LES, the well-known Smagorinsky [30] model 
is used to consider the influence of small scale eddies and to calculate the subgrid scale tensor. All computations are carried out with a Smagorinsky constant of Cs=0.1, which is an empirical value mostly used for practical applications [31].

Moreover, a volume-averaged method (VAM) is employed to describe the macroscopic flow in porous regions by modifying the Navier-Stokes equations [32], with an additional source term added on the right-hand side of the momentum equation to represent the pressure drop in the porous medium. The viscous and inertial resistances of the porous zone are described by the following Ergun's equation [33]:

$$
|\nabla P|=\frac{150 \mu}{d_{p}^{2}} \frac{(1-\varepsilon)^{2}}{\varepsilon^{3}} U+\frac{1.75}{d_{P}} \frac{(1-\varepsilon)}{\varepsilon} \rho_{0} U^{2}
$$

where $d_{p}$ and $\varepsilon$ are the mean particle diameter and porosity, respectively, and $U$ is the superficial velocity. This method has been widely used to study the flow characteristics of porous zones, such as Bhattacharyya [34] and Naito [35].

Different spatial and temporal discretization schemes are used for the governing equations, in which spatial discretization is achieved by using the bounded central differencing scheme for the convection terms and second-order central difference for the diffusion terms, and an implicit second-order scheme is utilized for the temporal discretization.

The computational domain is discretized with hexahedral meshes and the total number of mesh cells is around 5.4 million, as shown in Fig. 2. The grids are highly clustered in the vicinity of the cylinder surface and in the near wake including the porous treatment region, and the normal distance between the first layer mesh nodes and the cylinder surface is set to be $0.004 \mathrm{~mm}$, with a stretching factor of 1.08 . Moreover, 64 and 240 grids are distributed uniformly along the spanwise and circumferential directions, respectively, which are similar to the grid numbers and distributions utilized in previous simulations [29, 36, 37]. The above grid distribution ensures 
that the non-dimensional nearest wall distance $y^{+}$is smaller than 1 . Fig. 3 reports the distribution of $y^{+}$, which satisfies the requirement for the LES.

Time step $\Delta t=5 \times 10^{-5} \mathrm{~s}$ for the current simulation is used to ensure temporal resolution of the numerical result, which corresponds to the non-dimensional parameter $U_{\infty} \Delta t / D=0.004$, as suggested by Manoha et al.[38].
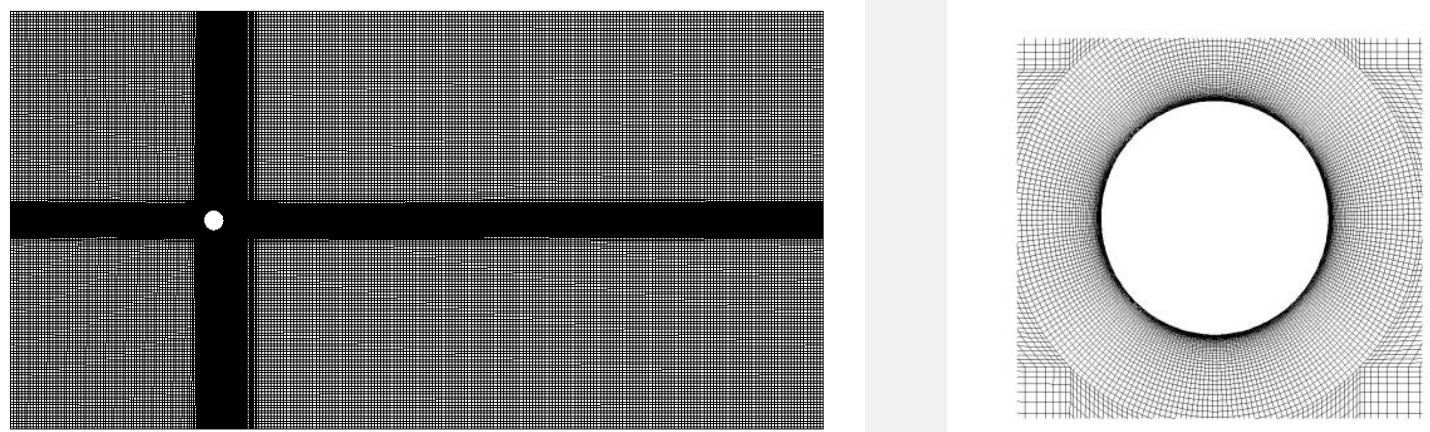

(a)

(b)

Fig. 2 Computational mesh for the circular cylinder: (a) whole domain; (b) region near the wall

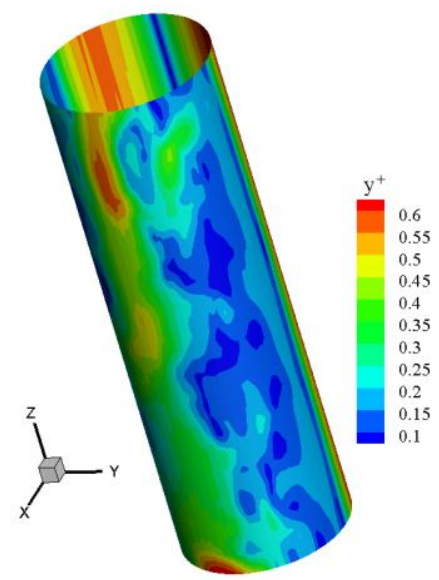

Fig. $3 y^{+}$distribution for the circular cylinder 


\subsection{Numerical validation and discussion}

For the flow past a smooth circular cylinder, both the time-averaged and fluctuating quantities calculated from the present simulation are compared with existing numerical and experimental results. Fig. 4 presents the time-averaged pressure coefficient along the cylinder surface, which is defined by

$$
C p=\frac{p-p_{\infty}}{0.5 \rho_{\infty} U_{\infty}{ }^{2}}
$$

where $p_{\infty}$ and $\rho_{\infty}$ are the reference pressure and density, respectively, which are chosen as the incoming flow values. The present numerical result is in good agreement with the experimental data of Norberg [25] and has slight deviations from the numerical results obtained by Mittal [39] and Breuer[26] and Liyes[40].

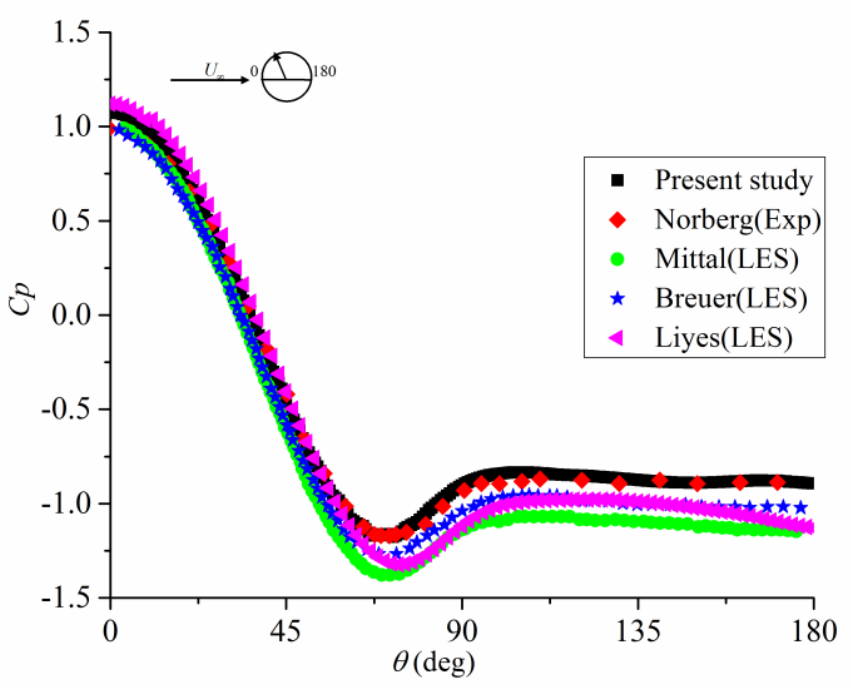

Fig. 4 Time-averaged pressure coefficient on the wall surface of the circular cylinder

As shown in Fig. 5, the time-averaged streamwise velocity $\bar{U}_{x}$ along the centreline of the cylinder wake is compared with experimental results of Lourenco and Shih [41] and Parnaudeau et al.[42] as well as numerical results of Meyer[27], Kahll[43] and Liyes[40]. All experimental 
measurements and numerical simulations reveal following features of the flow development downstream the cylinder. In the wake region very close to the cylinder $(\mathrm{x} / D<1)$, the mean flow velocity is negative and decreases with the flow development due to shed vortices in the recirculation zone, as shown in Fig. 5. In the region of $1<\mathrm{x} / D<2$, the mean flow velocity reaches the minimum and then recovers as the flow development further downstream. We can find the deficit velocity in this region is very large, implying that a strong shear stress and momentum exchange exist in this region. With the further development of the wake, the mean flow velocity $\bar{U}_{x}$ gradually recovers and approaches to the same order of the upstream mean flow velocity.

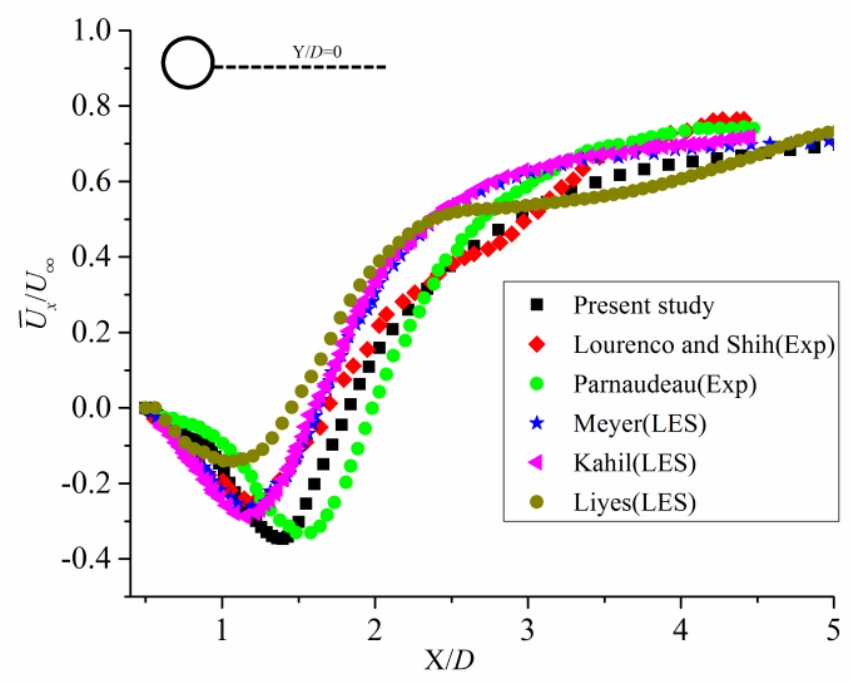

Fig. 5 Time-averaged streamwise velocity along the centerline at $y=0$

However, results from different studies do not collapse with each other, indicating that the streamwise velocity is very sensitive to experimental and numerical techniques. For experimental measurements, many factors, such as upstream disturbances and smoothness of the cylinder surface, can greatly affect the flow transition on the boundary layer and subsequent flow separation and development. For numerical simulations, it is still a challenge to accurately 
simulate the flow development in the near wake region with turbulence models due to the low turbulence viscosity in this region. For example, Breuer [26] illustrated that discretization schemes, mesh distributions and subgrid models had a great influence on the prediction of the length of the recirculation zone.

Fig. 6 displays time-averaged velocity contours and streamlines at the surface of $\mathrm{z}=0$. The result further verifies that the low velocity zone downstream the circular cylinder is caused by the vortex shedding from the cylinder surface.

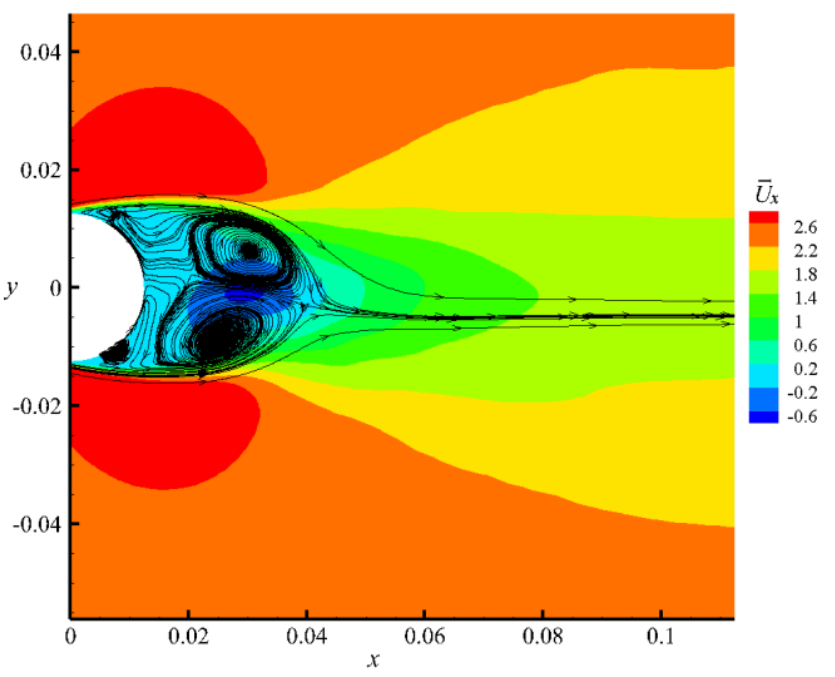

Fig. 6 Time-averaged velocity contours at the surface of $\mathrm{z}=0$

Time-averaged streamwise velocity profiles at different downstream locations $(\mathrm{x} / D=0.58$, 1.06, 1.54, 2.02) are displayed in Fig. 7 . At $\mathrm{x} / D=0.58$, the streamwise velocity profile calculated from the present simulation is in good agreement with the previous experimental data of Parnaudeau et al.[42] and Lourenco and Shih [41] as well as numerical results of Meyer[27] and Liyes [40]. At downstream positions of $\mathrm{x} / D=1.06,1.54$ and 2.02, all experimental and numerical results indicate that both the maximum deficit velocity and velocity gradient reduce with the 
wake development, but the detailed recovery of the deficit velocity has some deviations among all the data.

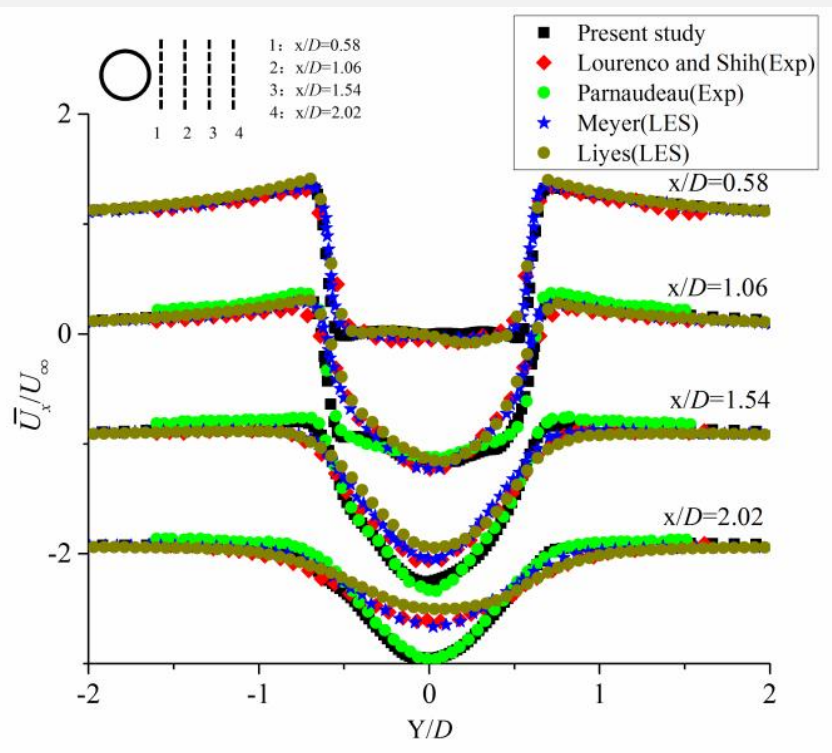

Fig. 7 Mean flow velocity at different downstream locations

Moreover, previous experimental and numerical studies have reported the time-averaged and root-mean square (RMS) values of lift and drag coefficients as well as the Strouhal number, and some of these data are listed in Table 1. Theoretically, the time-averaged lift coefficient should be equal to zero, and the present numerical and previous results show a consistency with this value. The time-averaged drag coefficient of collected experimental and numerical results ranges from 0.99 to 1.16 , and the present numerical result is 1.09 , indicating that the present simulation result is reasonable. The Strouhal number of the present simulation is 0.209 , which also reaches a good agreement with previous experimental and numerical results ranging from 0.202 to 0.223 .

On the RMS of the lift and drag coefficients, previous experimental and numerical studies suggest that their value are very sensitive to some factors. For instance, experimental results of Norberg [44] illustrate that the RMS lift coefficient changes significantly from 0.03 to 0.1 for 
Reynolds number in the range from 3000 to 5000, and numerical studies of Ouvrard [45] show that, even at $\mathrm{Re}=3900$, the calculated RMS lift coefficient varies from 0.051 to 0.060 and RMS drag coefficient fluctuates from 0.014 to 0.072 , and this is affected by the SGS model used in LES and the grid resolution. Therefore, the RMS lift and drag coefficient is very sensitive at the subcritical Reynolds number. In the present simulation, the RMS lift and drag coefficients are 0.274 and 0.025 , respectively, both of which are reasonable compared with existing results.

Table 1 Comparison of main flow quantities

\begin{tabular}{|l|c|c|c|c|c|}
\hline & $\bar{C}_{d}$ & $C_{d r m s}$ & $\bar{C}_{l}$ & $C_{l r m s}$ & $S t$ \\
\hline Present results & 1.09 & 0.025 & $-2.7 \times 10^{-3}$ & 0.274 & 0.209 \\
\hline Lourenco and Shih [41] & $0.98 \pm 0.05$ & - & - & - & $0.215 \pm 0.005$ \\
\hline Norberg [46] & $0.99 \pm 0.05$ & - & - & 0.09 & - \\
\hline Breuer [26] & 1.099 & - & - & & - \\
\hline Kahil [39] & 1.02 & 0.033 & $-8 \times 10^{-5}$ & 0.137 & 0.207 \\
\hline Frolich [47] & $0.99 \sim 1.02$ & $0.022 \sim 0.044$ & - & $0.052 \sim 0.3$ & 0.223 \\
\hline Ouvrard [45] & 1.16 & $0.014 \sim 0.072$ & - & $0.051 \sim 0.604$ & 0.212 \\
\hline Liyes [40] & 1.158 & 0.099 & $8 \times 10^{-4}$ & 0.316 & 0.202 \\
\hline
\end{tabular}

\section{Results and analysis}

In this section, the effect of the downstream porous material on the wake development as well as fluctuations of the lift and drag are studied. Hereafter the flow past the circular cylinder is named Case 1, and the flow past the circular cylinder and through the downstream porous material is named Case 2. 


\subsection{Time-averaged flow field}

The velocity distributions at four planes are compared to analyse the effect of porous material on the development of the wake mean flow. As shown in Fig. 8, Planes $1(\mathrm{x} / D=0.58)$ and 2 $(\mathrm{x} / D=1.06)$ are located in the region between the cylinder and the porous material, Planes 3 $(\mathrm{x} / D=1.54)$ is located inside the porous region $(1.5<\mathrm{x} / D<1.9)$ and Plane $4(\mathrm{x} / D=2.02)$ is downstream the porous material.

The velocity profiles at Plane 1 are nearly identical between these two cases, indicating that the porous material has little effect on the mean flow of the initial wake. At Plane 2, Fig. 8(a) shows that the maximum deficit velocity is the same for these two cases, but the wake width in Case 2 is smaller than Case 1. Additionally, Fig. 8(b) shows that the transverse velocity becomes larger in Case 2, implying that the porous material is beneficial to enhance the momentum exchange between the free stream and the wake. At Plane 3 which is inside the porous material, not only the maximum deficit velocity but also the wake width in Case 2 are greatly smaller than those in Case 1, and the transverse velocity in Case 2 is significantly larger than that in Case 1. Therefore, the above flow features reveal that the porous material can help to recover the mean flow in the wake due to the enhanced momentum exchange between the wake and the free stream. Furthermore, the deficit velocity at Plane 4 almost disappears at Case 2 as shown in Fig. $8(a)$, thus the momentum exchange becomes very weak and the transverse velocity is very small as shown in Fig. 8(b). 


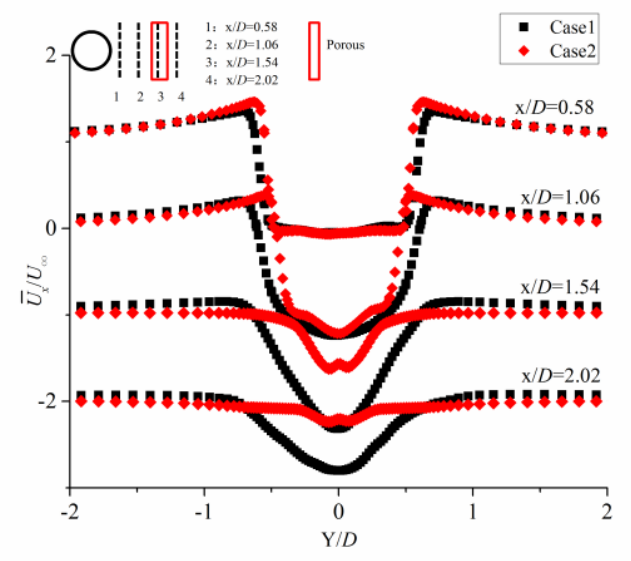

(a)

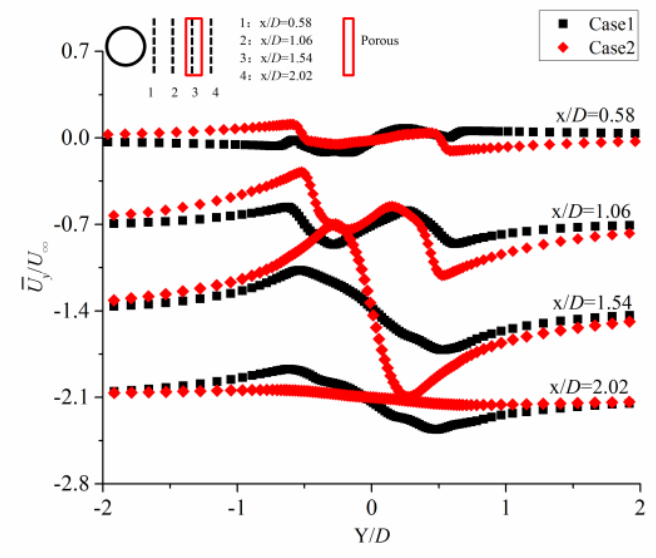

(b)

Fig. 8 Mean streamwise velocity at different locations in the wake of a circular cylinder with porous and without porous: (a) $\bar{U}_{x} / U_{\infty}$; (b) $\bar{U}_{y} / U_{\infty}$

In addition, the separation angle can be determined based on the time-averaged mean flow. In Case 1 , the separation angle $89.03^{\circ}$ obtained from present numerical simulation is consistent with the values presented in literatures of Ma et al.[48], Meyer[27] and Lourenco and Shih [41]. In Case 2, the separation angle is 90.23 , which is very close to that in Case 1, implying that the porous material actually does not affect the time-averaged flow around the cylinder surface. This conclusion is consistent with the results shown in Fig. 8, in which the time-averaged velocity profiles at surfaces of $\mathrm{x} / D=0.58$ and $\mathrm{x} / D=1.06$ are nearly the same for Case 1 and Case 2 .

The normalized Reynolds normal stresses $\overline{u^{\prime 2}} / U_{\infty}{ }^{2}, \overline{v^{\prime 2}} / U_{\infty}{ }^{2}$ and Reynolds shear stress $\overline{u^{\prime} v^{\prime}} / U_{\infty}^{2}$ in the near wake region of the circular cylinder $(\mathrm{x} / D=0.58,1.06,1.54,2.02)$ are presented in Fig. 9(a)-(c), respectively. At Case 1, the Reynolds normal stress $\overline{u^{\prime 2}} / U_{\infty}^{2}$ has two sharp peaks at the $\mathrm{x} / D=0.58$ and $\mathrm{x} / D=1.06$ mainly due to the transitional state of the shear layers shown in Fig. 9(a). Concerning the Reynolds normal stress $\overline{v^{\prime 2}} / U_{\infty}{ }^{2}$, Fig. 9(b) indicates that it is 
nearly symmetrical to the wake centerline and the maximum value occurs at the centerline. Fig. 9(c) illustrates that the Reynolds shear stress $\overline{u^{\prime} v^{\prime}} / U_{\infty}{ }^{2}$ is antisymmetric. All these flow features are consistent with numerical simulations of Meyer [27]. For Case 2, all the profiles of the above-mentioned second order statistics are much smaller than that of Case 1, indicating that the porous material can greatly suppress both the turbulence kinetics and the Reynolds shear stress.

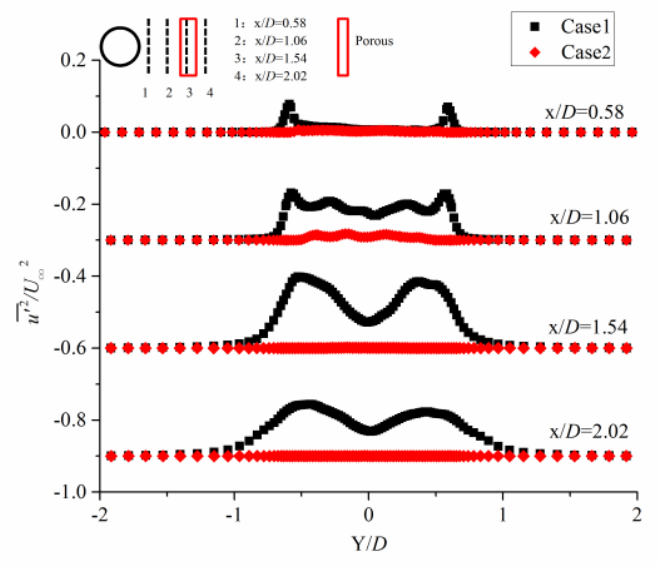

(a)

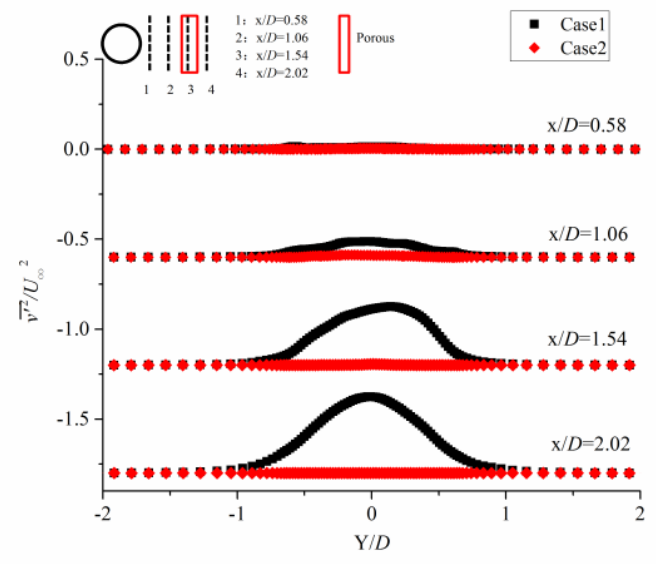

(b)

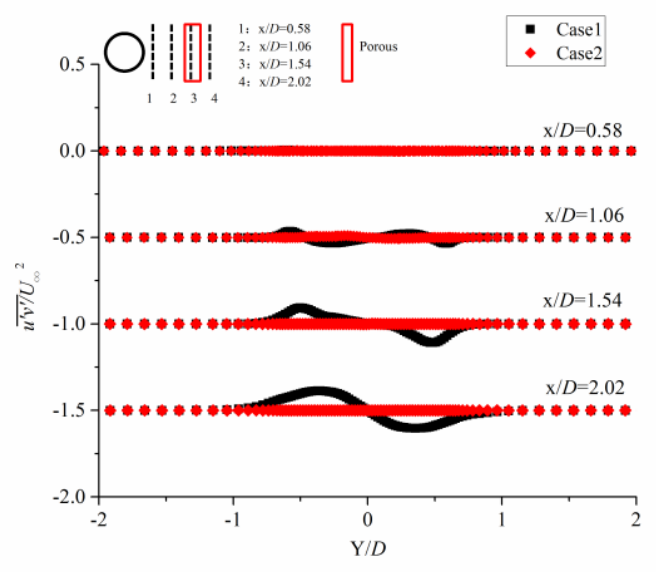

(c)

Fig. 9 Reynolds stress in the wake region: (a) $\overline{u^{\prime 2}} / U_{\infty}^{2}$; (b) $\overline{v^{\prime 2}} / U_{\infty}^{2}$; (c) $\overline{u^{\prime} v^{\prime}} / U_{\infty}^{2}$

Based on the preceding comprehensive analysis, we can conclude that effects of the applied porous material on mean and fluctuating flow fields are different. The porous material greatly 
reduces the gradient of the time-averaged flow inside and downstream it, but the porous material does not affect the time-averaged flow upstream it. On the other hand, the porous material enables to significantly reduce both the turbulence kinetics and the Reynolds shear stress in the whole flow field downstream the cylinder, thus we can deduce that the off-body porous material reduces the turbulence viscosity $v_{T}$, which is defined by

$$
-\overline{u^{\prime} v^{\prime}}=v_{T} \frac{\partial U}{\partial y}
$$

\subsection{Instantaneous flow field}

In order to get further insights into the effect of the off-body porous treatment on the flow around the circular cylinder, instantaneous contours of the velocity magnitude at $\mathrm{z}=0$ plane are plotted for the cases with and without porous treatment in Fig. 10. As expected, Fig. 10(a) shows a low velocity zone downstream the circular cylinder as well as alternating shedding vortices. However, when the porous material is fixed downstream of the cylinder, Fig. 10(b) shows that the flow mode is totally different from that of the cylinder without porous treatment. Although low velocity zone still exists, alternating shedding vortices have been replaced by symmetric shedding vortices. As we know, laminar separation from the cylinder will induce two symmetric shedding vortices[49]. Therefore, we can speculate that the flow feature in Fig. 10(b) is laminar as well. Both the instantaneous vortices and subgird viscosity ratio are used to further confirm the flow status shown in Fig. 10(b).

The instantaneous vortex based on the definition of Q criterion [50] is depicted in Fig. 11. Formation and development of vortices starting from the cylinder surface are visual in Fig. 11(a), but Fig. 11(b) indicates that nearly no vortex structures can be identified. 

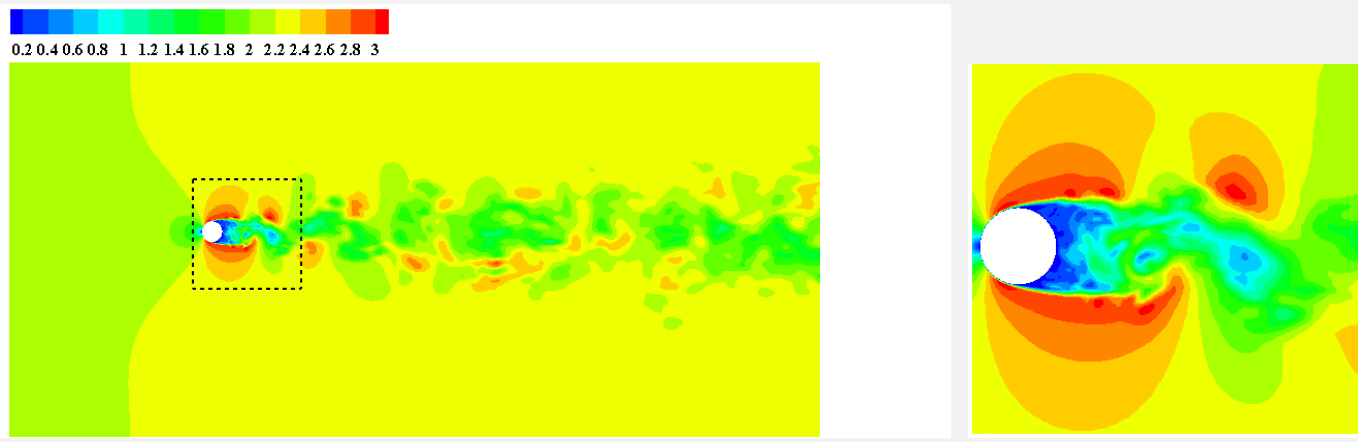

(a)
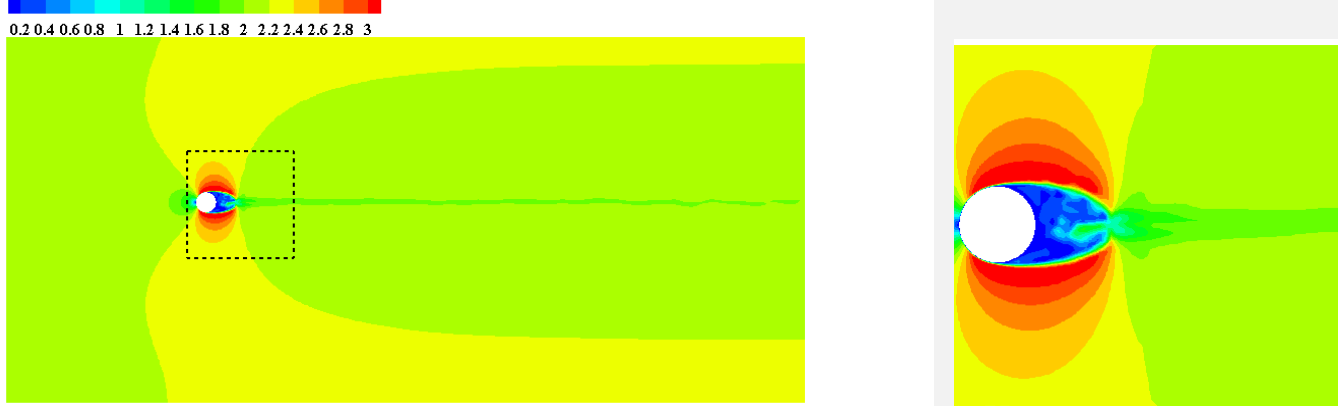

(b)

Fig. 10 Instantaneous contours of the velocity magnitude at $\mathrm{Z}=0$ plane: (a) Case1; (b) Case 2
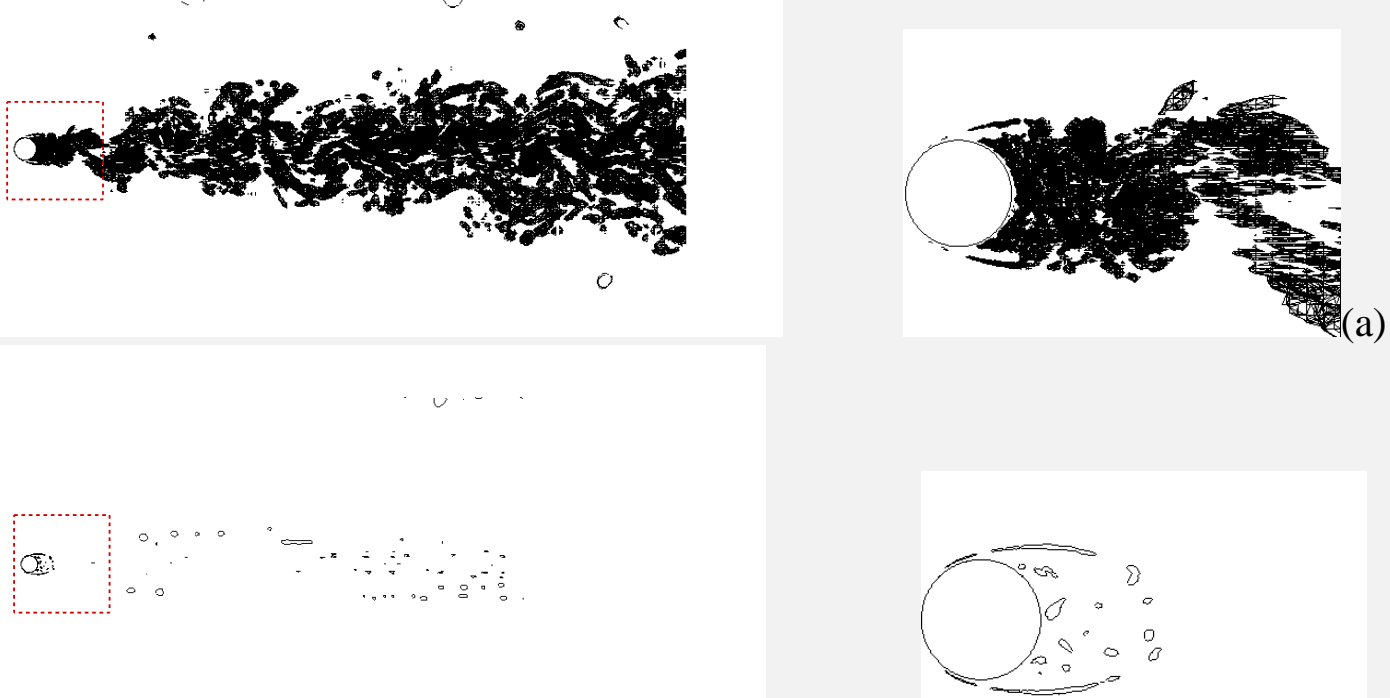

(b)

Fig. 11 Instantaneous contours of $\mathrm{Q}=0.2$ : (a) Case1; (b) Case2

Contours of the subgrid viscosity ratio at $\mathrm{z}=0$ plane are displayed in Fig. 12. Fig. 12(a) indicates the subgrid viscosity ratio inside the wake region is much larger than that outside the wake region, implying relatively strong turbulent features of free developed wake. However, the 
subgrid viscosity ratio shown in Fig. 12(b) nearly approaches to zero, implying that the SGS model in the present simulation is actually inactive and the flow is almost laminar.
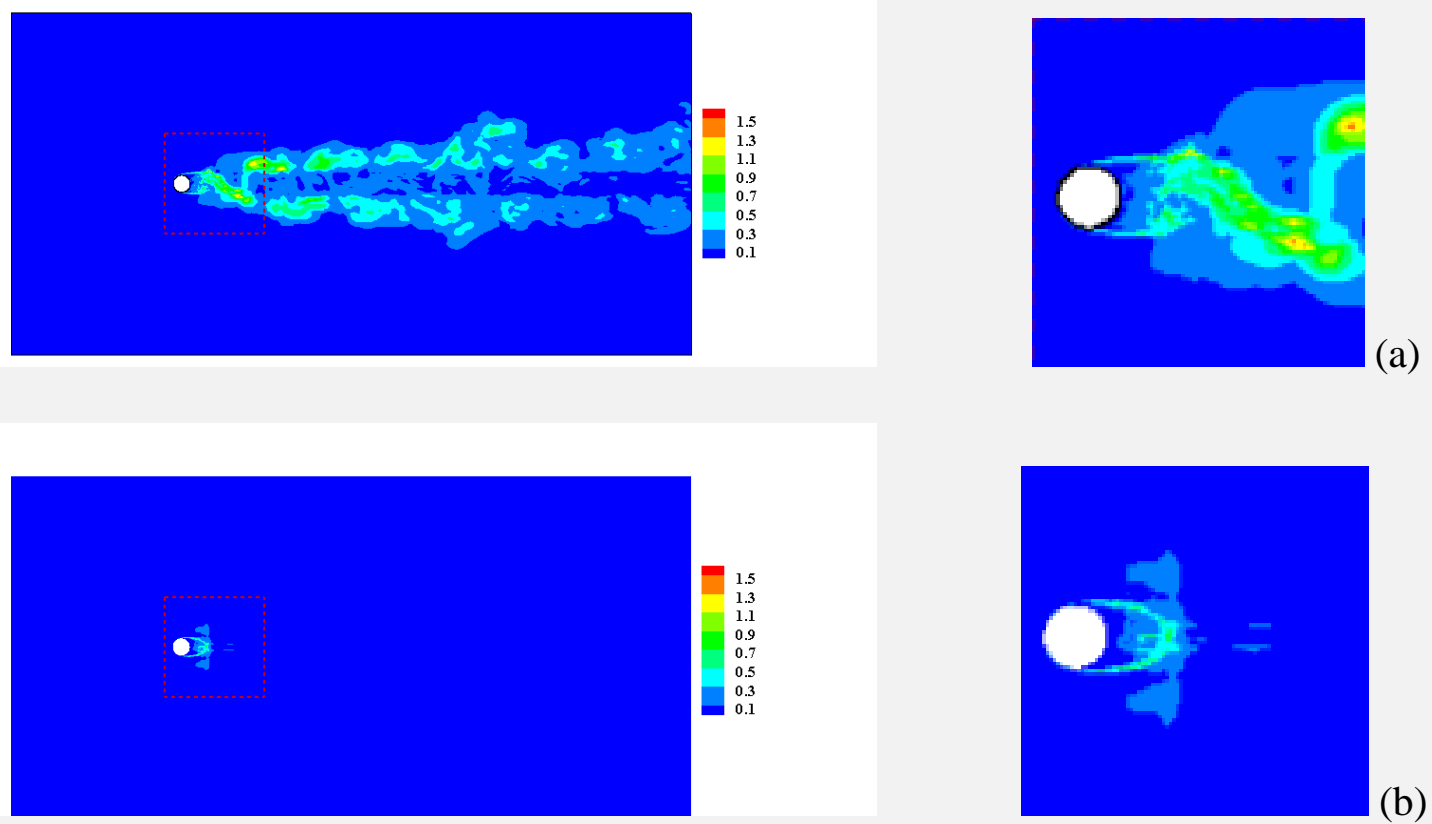

Fig. 12 Subgird viscosity ratio at $\mathrm{z}=0$ plane: (a) Case 1; (b) Case 2

All the above features confirm that the flow with two symmetric steady separation bubbles is laminar. Owing to laminarization of the cylinder flow caused by the downstream porous material, we now can easily understand why profiles of the time-averaged velocity are nearly the same in these two cases but the turbulence intensity in Case 1 is much higher than that of Case 2. Moreover, the computational result from Case 2 also indicates that the time-averaged total pressure drop caused by the porous material is merely about $7 \mathrm{~Pa}$, and this flow loss can be negligible compared with the pressure drop caused by the vortex shedding from the circular cylinder.

Another benefit contributed from the laminarization is that fluctuations of the lift and drag are greatly reduced, as shown in Fig. 13. At Case 1, periodic lift fluctuation can be observed due to alternating vortex shedding, but fluctuations of the lift and drag are very small at Case 2 due to 
the nearly steady flow separation. Moreover, Fig. 14 compares spectra of the lift coefficient for these two cases. The result shows the vortex shedding frequency is $f_{\mathrm{s}}=18.33 \mathrm{~Hz}$ at Case 1 , which is in good agreement with previous studies (Strouhal number $=0.209 \pm 0.001$ ) listed in Table 1 . At Case 2, not only the magtitude of lift coefficient is very small but also no singnificant peaks can be found in the spectrum.

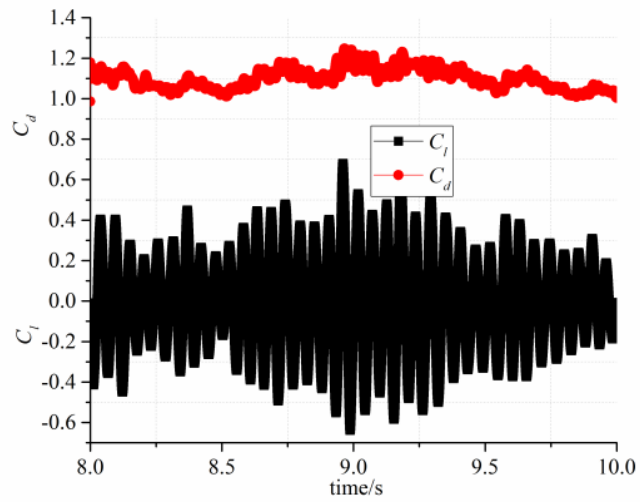

(a)

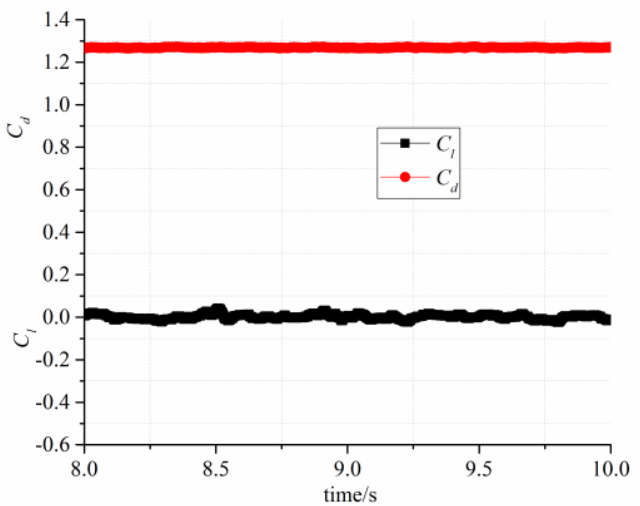

(b)

Fig. 13 Time histories of lift and drag coefficients: (a) Case 1; (b) Case 2

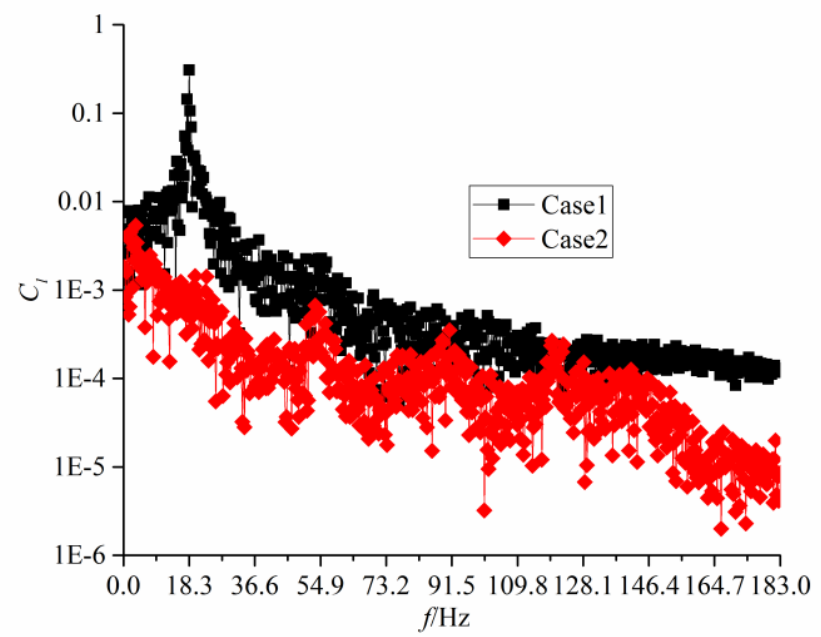

Fig. 14 Spectra of lift coefficients

Fig. 15 plots the power spectral density of the transverse velocity at the centerline of the wake at four downstream planes of $\mathrm{x} / D=0.58,3,5,10$, covering a wide range from near wake to far 
wake. At Case 1, the peak values of the spectra are very noticeable at the normalized frequency $f \mid f_{\mathrm{s}}=1$, due to the alternative vortex shedding from the cylinder surface. However, amplitudes of fluctuating velocities are very small and no peaks can be found for Case 2, further confirming that the flow is laminar.

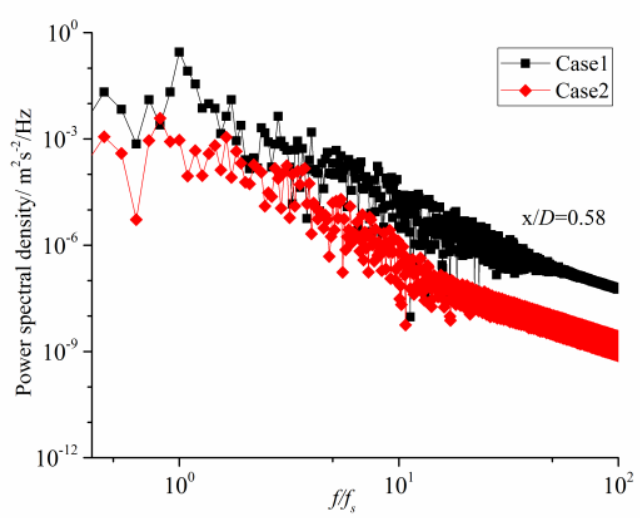

(a)

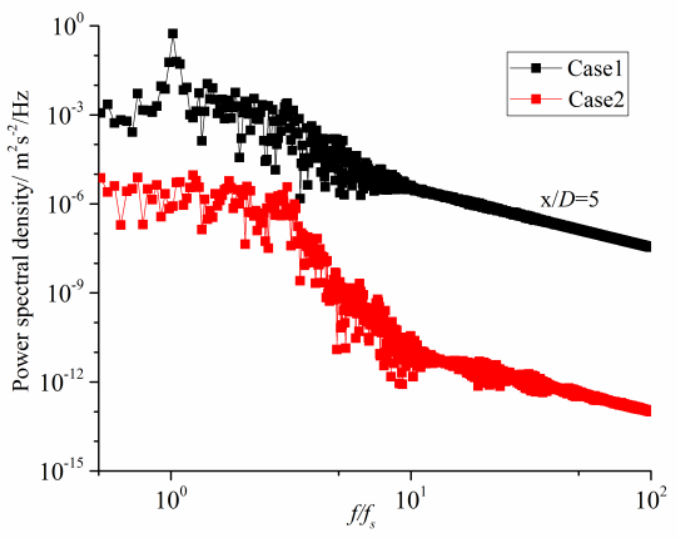

(c)

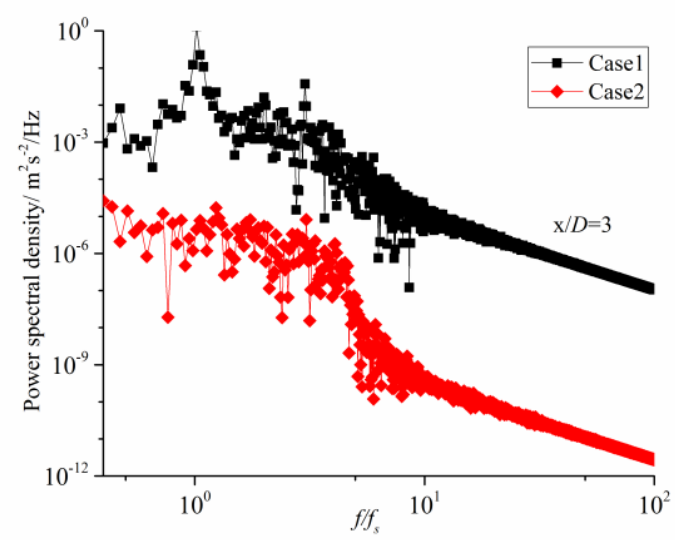

(b)

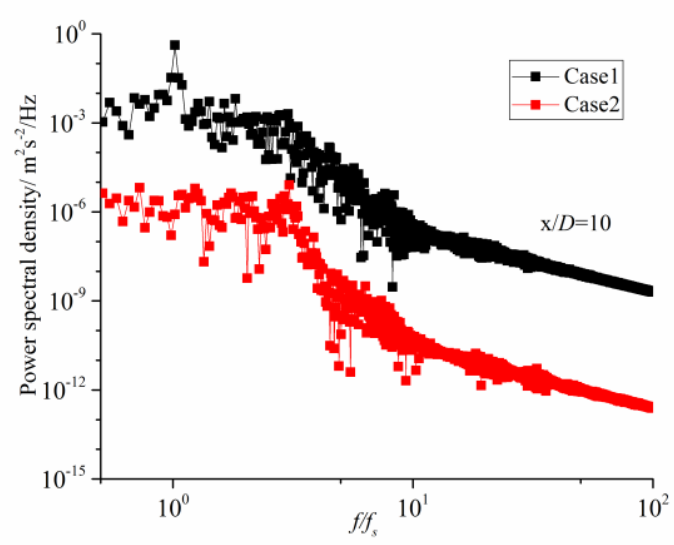

(d)

Fig. 15 Power spectral density of transverse velocity: (a) $\mathrm{x} / D=0.58$; (b) $\mathrm{x} / D=3$; (c) $\mathrm{x} / D=5$; (d)

$$
\mathrm{x} / D=10
$$

\subsection{Aerodynamic noise}

The acoustic analogy proposed by Lighthill [51] and further developed by Curle [52] is used to calculate aerodynamic noise radiated from flow and its interaction with the solid surface. As 
the flow Mach number is very small, noise contributed from quadrupole sources is much smaller than that from dipole sources, thus only the noise radiated from pressure fluctuations acting on the stationary solid surface is calculated with the Curle's equation [52].

Fig. 16 displays RMS of the wall surface pressure fluctuations. The RMS value of the Case 1 is much larger than that of the Case 2, implying that noise generated from the Case 2 should be greatly lower than that from the Case 1 .

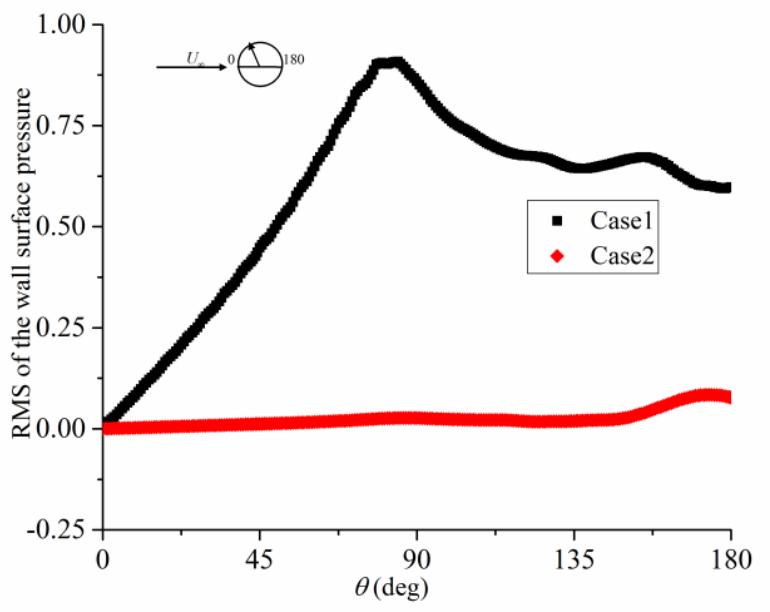

Fig. 16 RMS of the wall surface pressure fluctuations

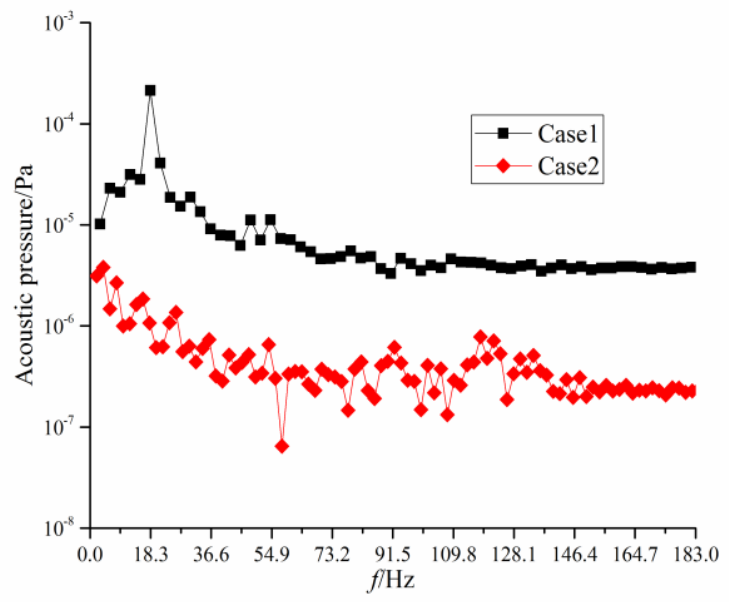

Fig. 17 Acoustic pressure spectra for noise radiated form dipole sources on the cylinder surface 
Fig. 17 displays the sound pressure spectra of the observer at the position of $(0,1 \mathrm{~m}, 0)$. It can be seen that the maximum value of the acoustic pressure for Case 1 occurs at vortex shedding frequency of $18.3 \mathrm{~Hz}$, which is consistent with that of the lift coefficient shown in Fig. 14. Compared with Case 1, the acoustic pressure of Case 2 has a great reduction over the entire range of frequencies.

The research confirms that noise level can be greatly suppressed by fixing an off-body porous material downstream of the circular cylinder. It should be noted that this conclusion is only drawn from the present numerical simulation. As a potential method for controlling flow separation from blunt bodies and associated noise generation, more parametric studies, such as the porosity and downstream distance of the porous material as well as the flow Reynolds number, should be performed to test the its robustness and applicable range.

\section{Conclusions}

An off-body based passive flow control method is developed to suppress unsteady flow separation and the vortex shedding from a circular cylinder by placing the porous material downstream the cylinder. The following conclusions are drawn from the numerical study.

(1) At the subcritical Reynolds number $\mathrm{Re}=3900$ studied in this paper, the unsteady turbulence vortex shedding from the cylinder surface is transmitted into the steady laminar vortex shedding by fixing the porous material downstream the circular cylinder, thus the porous material achieves a reverse transition from turbulence to laminar flow.

(2) Laminarization caused by the downstream porous material has little effect on the timeaveraged wake mean flow in the region close to the cylinder surface as well as the time-averaged flow drag, thus the downstream porous material is not a good choice to reduce the time-averaged flow drag. 
(3) Laminarization caused by the downstream porous material greatly suppresses the lift and drag fluctuations, the turbulence kinetic energy and the noise level, thus the downstream porous material can be used as an efficient method to control the unsteady flow as well as the associated noise.

The present study initials an off-body based passive flow control method and shows promising potential in engineering applications, it is planned to carry out further investigations on the following two aspects:

(1) It is necessary to deepen the understanding of the flow control mechanism, i.e., the physical mechanism of reverse transition from turbulence to laminar flow caused by the porous material.

(2) Parametric studies, such as the geometry and position of the porous material as well as the Reynolds number of the mean flow, are meaningful for applications in various engineering fields.

\section{Acknowledgments}

The research is supported by the National Natural Science Foundation of China (Nos. 51806164, 51511130075) and International Postdoctoral Exchange Fellowship Program (No. 20160101). The authors gratefully acknowledge high-performance computing facilities IRIDIS5 at the University of Southampton in the completion of this work. Additionally, the authors would like to acknowledge the help of Dr. Abdallah S. Berrouk in Khalifa University of Science and Technology for providing some experimental and numerical data.

\section{References}

[1] M. Gad-el-Hak, Flow control, Applied Mechanics Reviews, 42 (1989) 261-293.

[2] M. Gad-el-Hak, Modern developments in flow control, Applied Mechanics Reviews, 49 (1996) 365-379. 
[3] L.G. Pack, R.D. Joslin, Overview of active flow control at NASA Langley Research Center, 5th Annual International Symposium on Smart Structures and Materials, San Diego, California, United States, 1998.

[4] L.N. Cattafesta, S. Garg, D. Shukla, Development of piezoelectric actuators for active flow control, AIAA Journal, 39 (2001) 1562-1568.

[5] L. Cattafesta, F. Alvi, D. Williams, C. Rowley, Review of active control of flow-induced cavity oscillations, 33rd AIAA Fluid Dynamics Conference and Exhibit, Orlando, Florida, United States, 2003.

[6] I. Gursul, Z. Wang, E. Vardaki, Review of flow control mechanisms of leading-edge vortices, Progress in Aerospace Sciences, 43 (2007) 246-270.

[7] P. Zhang, J. Wang, L. Feng, Review of zero-net-mass-flux jet and its application in separation flow control, Science in China series E: technological sciences, 51 (2008) 1315.

[8] T.C. Corke, C.L. Enloe, S.P. Wilkinson, Dielectric barrier discharge plasma actuators for flow control, Annual Review of Fluid Mechanics, 42 (2010) 505-529.

[9] L.N. Cattafesta III, M. Sheplak, Actuators for active flow control, Annual Review Fluid of Mechanics, 43 (2011) 247-272.

[10] C. Seal, C. Smith, The control of turbulent end-wall boundary layers using surface suction, Experiments in Fluids, 27 (1999) 484-496.

[11] J. Kim, H. Choi, Distributed forcing of flow over a circular cylinder, Physics of Fluids, 17 (2005) 033103.

[12] J. Tensi, I. Boué, F. Paillé, G. Dury, Modification of the wake behind a circular cylinder by using synthetic jets, Journal of Visualization, 5 (2002) 37-44. 
[13] Y.G. Liu, L.H. Feng, Suppression of lift fluctuations on a circular cylinder by inducing the symmetric vortex shedding mode, Journal of Fluids and Structures, 54 (2015) 743-759.

[14] M.L. Post, T.C. Corke, Separation control on high angle of attack airfoil using plasma actuators, AIAA Journal, 42 (2004) 2177-2184.

[15] G. Nati, M. Kotsonis, S. Ghaemi, F. Scarano, Control of vortex shedding from a blunt trailing edge using plasma actuators, Experimental Thermal and Fluid Science, 46 (2013) 199210.

[16] A. Ebrahimi, M. Hajipour, K. Ghamkhar, Experimental study of stall control over an airfoil with dual excitation of separated shear layers, Aerospace Science and Technology, 82 (2018) $402-411$.

[17] K. Kwon, H. Choi, Control of laminar vortex shedding behind a circular cylinder using splitter plates, Physics of Fluids, 8 (1996) 479-486.

[18] J.Y. Hwang, K.S. Yang, S.H. Sun, Reduction of flow-induced forces on a circular cylinder using a detached splitter plate, Physics of Fluids, 15 (2003) 2433-2436.

[19] R. Pérez-Torró, J.W. Kim, A large-eddy simulation on a deep-stalled aerofoil with a wavy leading edge, Journal of Fluid Mechanics, 813 (2017) 23-52.

[20] N. Thomareis, G. Papadakis, Numerical analysis of a trailing edge with triangular serrations using dynamic mode decomposition, 54th AIAA Aerospace Sciences Meeting, San Diego, California, United States, 2016.

[21] B. Gozmen, E. Firat, H. Akilli, B. Sahin, Flow control behind a circular cylinder via a porous cylinder in deep water, EPJ Web of Conferences, 45(2013) 01035.

[22] T. Sueki, T. Takaishi, M. Ikeda, N. Arai, Application of porous material to reduce aerodynamic sound from bluff bodies, Fluid Dynamics Research, 42 (2010) 015004. 
[23] H. Liu, J. Wei, Z. Qu, Prediction of aerodynamic noise reduction by using open-cell metal foam, Journal of Sound Vibration, 331 (2012) 1483-1497.

[24] H.R. Liu, M. Azarpeyvand, J.J. Wei, Z.G. Qu, Tandem cylinder aerodynamic sound control using porous coating, Journal of Sound Vibration, 334 (2015) 190-201.

[25] C. Norberg, Effects of Reynolds number and a low-intensity freestream turbulence on the flow around a circular cylinder, Chalmers University, Goteborg, Sweden, Technological Publications, 2 (1987) 1-55.

[26] M. Breuer, Large eddy simulation of the subcritical flow past a circular cylinder: numerical and modeling aspects, International Journal for Numerical Methods in Fluids, 28 (1998) 12811302.

[27] M. Meyer, S. Hickel, N.A. Adams, Assessment of Implicit Large-Eddy Simulation with a Conservative Immersed Interface Method for turbulent cylinder flow, International Journal of Heat and Fluid Flow, 31 (2010) 368-377.

[28] C. Xu, Y.J. Mao, Experimental investigation of metal foam for controlling centrifugal fan noise, Applied Acoustics, 104 (2016) 182-192.

[29] D.A. Lysenko, I.S. Ertesvåg, K.E. Rian, Large-eddy simulation of the flow over a circular cylinder at reynolds number 3900 using the openfoam toolbox, Flow, Turbulence and Combustion, 89 (2012) 491-518.

[30] J. Smagorinsky, General circulation experiments with the primitive equations: I. The basic experiment, Monthly Weather Review, 91 (1963) 99-164.

[31] R.S. Rogallo, P. Moin, Numerical simulation of turbulent flows, Annual Review of Fluid Mechanics, 16 (1984) 99-137. 
[32] K. Vafai, Convective flow and heat-transfer in variable-porosity media, Journal of Fluid Mechanics, 147 (1984) 233-259.

[33] S. Ergun, Fluid flow through packed columns, Chemical Engineering Progress, 48 (1952) 89-94.

[34] S. Bhattacharyya, A. Singh, Reduction in drag and vortex shedding frequency through porous sheath around a circular cylinder, International Journal for Numerical Methods in Fluids, 65 (2011) 683-698.

[35] H. Naito, K. Fukagata, Numerical simulation of flow around a circular cylinder having porous surface, Physics of Fluids, 24 (2012) 117102.

[36] S. Sarkar, Large-eddy simulation of wake and boundary layer interactions behind a circular cylinder, Journal of Fluids Engineering, 131 (2009) 1-13.

[37] M.Breuer, A challenging test case for large eddy simulation: high Reynolds number circular cylinder flow, International Journal of Heat and Fluid Flow, 21 (2000) 648-654

[38] E. Manoha, B. Troff, P. Sagaut, Trailing-edge noise prediction using large-eddy simulation and acoustic analogy, AIAA Journal, 38 (2000) 575-583.

[39] R. Mittal, Progress on LES of flow past a circular cylinder, Center for Turbulence Research Annual Research Briefs, 1996 233-241.

[40] L. Aguedal, D. Semmar, A.S. Berrouk, A. Azzi, H. Oualli, 3D vortex structure investigation using Large Eddy Simulation of flow around a rotary oscillating circular cylinder, European Journal of Mechanics - B/Fluids, 71 (2018) 113-125.

[41] C.S. L. Lourenco, Characteristics of the plane turbulent near wake of a circular cylinder, a particle image velocimetry study Private Communication, 1993. 
[42] P. Parnaudeau, J. Carlier, D. Heitz, E. Lamballais, Experimental and numerical studies of the flow over a circular cylinder at Reynolds number 3900, Physics of Fluids, 20 (2008) 085101. [43] Y.Kahil, Large eddy simulation of the turbulent flows around circular cylinders at subcritical reynolds number (Ph.D thesis), Paris, France, 2011 6-7.

[44] C. Norberg, Flow around a circular cylinder: aspects of fluctuating lift, Journal of Fluids and Structures, 15 (2001) 459-469.

[45] H. Ouvrard, B. Koobus, A. Dervieux, M.V. Salvetti, Classical and variational multiscale LES of the flow around a circular cylinder on unstructured grids, Computers \& Fluids, 39 (2010) 1083-1094.

[46] C. Norberg, An experimental investigation of the flow around a circular cylinder: influence of aspect ratio, Journal of Fluid Mechanics, 258 (1994) 287-316.

[47] J. Fröhlich, W. Rodi, P. Kessler, S. Parpais, J. Bertoglio, D. Laurence, Large eddy simulation of flow around circular cylinders on structured and unstructured grids, Numerical Flow Simulation I, Springer, Berlin, Heidelberg, 1998 319-338.

[48] X. Ma, G.S. Karamanos, G.E. Karniadakis, Dynamics and low-dimensionality of a turbulent near wake, Journal of Fluid Mechanics, 410 (2000) 29-65.

[49] W.K. Blake, Mechanics of Flow-Induced Sound and Vibration, Volume 1: General Concepts and Elementary Source, Aplikace Matematiky, Applied Mathematics and mechanics, 17 (1986) 457.

[50] J.C. Hunt, A.A. Wray, P. Moin, Eddies, streams, and convergence zones in turbulent flows, Center for Turbulence Research Proceeding of the Summer Program, United States, 1988. 
[51] M.J. Lighthill, On Sound Generated Aerodynamically. I. General Theory, Proceedings of the Royal Society of London. Series A. Mathematical and Physical Sciences, 211 (1952) 564587.

[52] N. Curle, The influence of solid boundaries upon aerodynamic sound, Proceedings of the Royal Society of London Series-A, 231 (1955) 505-514. 